\title{
ARTICLE OPEN Charting the circuit QED design landscape using optimal control theory
}

\author{
Michael H. Goerz $\mathbb{D}^{1,4,5}$, Felix Motzoi ${ }^{2,3,6}$, K. Birgitta Whaley ${ }^{2,3}$ and Christiane P. Koch (iD) ${ }^{1}$
}

With recent improvements in coherence times, superconducting transmon qubits have become a promising platform for quantum computing. They can be flexibly engineered over a wide range of parameters, but also require us to identify an efficient operating regime. Using state-of-the-art quantum optimal control techniques, we exhaustively explore the landscape for creation and removal of entanglement over a wide range of design parameters. We identify an optimal operating region outside of the usually considered strongly dispersive regime, where multiple sources of entanglement interfere simultaneously, which we name the quasi-dispersive straddling qutrits regime. At a chosen point in this region, a universal gate set is realized by applying microwave fields for gate durations of $50 \mathrm{~ns}$, with errors approaching the limit of intrinsic transmon coherence. Our systematic quantum optimal control approach is easily adapted to explore the parameter landscape of other quantum technology platforms.

npj Quantum Information (2017)3:37 ; doi:10.1038/s41534-017-0036-0

\section{INTRODUCTION}

For quantum technology to unfold its full potential, ultimate performance bounds must be known. This concerns all relevant steps for operating a device, such as state preparation or quantum gate implementation. One such bound is the empirical "quantum speed limit" that determines the shortest possible duration to carry out the task at hand. ${ }^{1-3}$ Quantum optimal control $(\mathrm{QOC})^{4,5}$ has grown into a versatile tool for identifying these performance bounds. Typical control tasks include the preparation of nonclassical states, as shown in an experiment with a Bose-Einstein condensate, ${ }^{6}$ or the creation of entanglement and quantum error correction, as demonstrated with diamond spin systems. ${ }^{7,} 8$ To date, these tasks have been optimized for known, fixed parameters of the respective system. Here, we show that a fully numerical QOC approach can go even further and, using the most advanced control techniques, can map out the entire parameter landscape for the physical system at hand. To this end, we consider the task of realizing the fastest possible universal set of gates for two superconducting transmon qubits within the constraints of current lifetimes.

Superconducting transmon qubits ${ }^{9}$ are one of the most promising architectures for quantum computing today. The development of circuit $\mathrm{QED}^{10}$ a broad platform for quantum technology, in particular enabled the entanglement of spatially separated superconducting qubits via a shared transmission line resonator. The shared resonator can be used to implement twoqubit gates but is generally required to be decoupled when single-qubit gates are carried out.

Three principal approaches have been used to couple superconducting qubits via a resonator: fixed-frequency, ${ }_{1}^{11-20}$ tunable frequency, ${ }^{21-23}$ and tunable coupling. ${ }^{24-28}$ Fixed-frequency transmons require the least technological overhead but also make the realization of gates most difficult. Adding more overhead in terms of dedicated control lines for the purpose of qubit driving ${ }^{12}$ or frequency biasing ${ }^{9}$ can speed up gate implementation but comes at the cost of additional noise sources. ${ }^{9}, 29-31$

In all these approaches, a wide range of possible parameters can be engineered. However, the parameter regimes where single- and two-quit gates can be faithfully operated are typically very different and the optimal choice of parameters is then not obvious. Identification of the optimal operating parameters is a well-defined control problem that we address here with QOC. While QOC has been used to realize specific quantum gates on superconducting qubits, ${ }^{19,} 23,32-37$ no systematic exploration of the full parameter space has been undertaken to date. In particular, most prior work has focused exclusively on the dispersive regime and explicitly avoided the regime of strong coupling. We show here that a fully numerical approach combined with advanced QOC techniques allows us to map the entire parameter landscape without restrictions due to approximations or model reduction. We can thus identify the global quantum speed limit for a universal set of gates for transmon qubits and analyze how gate errors vary with qubit-cavity-couplings, resonances, and cavity-mediated decay. This guides the decision for specific working points that promise a successful implementation of universal quantum computing using superconducting qubits. In particular, our results show that fast operation of both the singleand two-qubit gates needed for universal computation can be implemented in parameter regimes outside those typically explored to date. The results thus provide critical information for design decisions in circuit QED, or similarly complex quantum architectures.

\footnotetext{
${ }^{1}$ Theoretische Physik, Universität Kassel, Heinrich-Plett-Strasse 40, D-34132 Kassel, Germany; ${ }^{2}$ Berkeley Center for Quantum Information and Computation, Berkeley, CA 94720, USA and ${ }^{3}$ Department of Chemistry, University of California, Berkeley, CA 94720, USA

Correspondence: Michael H. Goerz (goerz@stanford.edu)

${ }^{4}$ Present address: Edward L. Ginzton Laboratory, Stanford University, Stanford, CA 94305, USA

${ }^{5}$ Present address: U.S. Army Research Laboratory, Computational and Information Sciences Directorate, Adelphi, MD 20783, USA

${ }^{6}$ Present address: Department of Physics and Astronomy, Aarhus University, 8000 Aarhus C, Denmark
}

Received: 30 August 2016 Revised: 25 July 2017 Accepted: 30 July 2017

Published online: 18 September 2017 


\section{RESULTS}

Model and parameters

Two superconducting transmons with a common transmission line resonator can be modeled by two anharmonic ladders coupled to a driven harmonic oscillator. ${ }^{9}$ In the frame rotating with frequency $\omega_{r}$ and within the rotating wave approximation, the Hamiltonian reads

$$
\begin{aligned}
\hat{\mathrm{H}}= & \sum_{q=1,2} \hbar\left[\delta_{q} \hat{\mathrm{b}}_{q}^{\dagger} \hat{\mathrm{b}}_{q}+\frac{a_{q}}{2} \hat{\mathrm{b}}_{q}^{\dagger} \hat{\mathrm{b}}_{q}^{\dagger} \hat{\mathrm{b}}_{q} \hat{\mathrm{b}}_{q}+g\left(\hat{\mathrm{b}}_{q}^{\dagger} \hat{\mathrm{a}}+\hat{\mathrm{b}}_{q} \hat{\mathrm{a}}^{\dagger}\right)\right] \\
& +\hbar \delta_{c} \hat{\mathrm{a}}^{\dagger} \hat{\mathrm{a}}+\frac{\hbar}{2}\left(\epsilon(t) \hat{\mathrm{a}}+\epsilon^{*}(t) \hat{\mathrm{a}}^{\dagger}\right),
\end{aligned}
$$

with $\delta_{j}=\omega_{j}-\omega_{r}(j=1,2, c)$, where $\omega_{c}$ is the resonator ("cavity") frequency, $\omega_{1,2}$ is the frequency of the first (second) qubit, $a_{1,2}$ is the qubit anharmonicity, $g$ is the coupling strength between each qubit and the resonator, and $\epsilon(t)$ is the microwave control field in the cavity. Taking $\epsilon(t) \in \mathbb{C}$ is equivalent to controlling the pulse amplitude and phase independently. It corresponds to a control field $\epsilon(t) \in \mathbb{R}$ in the non-rotating frame whose driving frequency may deviate from $\omega_{r}$. In the following, we set $\hbar=1$.

Typically, the Hamiltonian (1) is treated perturbatively, in order to derive an effective model in which the cavity can be integrated out. This approach is only valid in the "dispersive regime" where the qubit-cavity separation is much larger than the qubit-cavity coupling, $\left|\omega_{c}-\omega_{1,2}\right| \gg g$. This limits the effective interaction $\propto \mathrm{g} /$ $\left(\omega_{c}-\omega_{1,2}\right)$ between both qubits, except when resonances with higher transmon levels can be exploited. ${ }^{14,15}$ Here, we forgo such a treatment in lieu of solving Eq. (1) numerically, allowing us to explore parameter regimes beyond the dispersive limit.

In order to limit the number of parameters that have to be varied, we focus on the two parameters that capture the essential physics of quantum gate implementation. The departure from the dispersive regime is characterized by $\Delta_{c} / g$, with $\Delta_{c}=\omega_{c}-\omega_{1}$. Secondly, resonances of the form $\left|\omega_{1}-\omega_{2}\right| \approx n a_{1,2}, n=1,2$, between different levels of the two transmons are known to aid in the implementation of entangling gates ${ }^{14,15}$; therefore, it is natural to express the qubit-qubit-detuning $\Delta_{2}=\omega_{2}-\omega_{1}$ in units of $a=\left|a_{1}+a_{2}\right| / 2$. We can thus explore the entire parameter landscape in terms of $\Delta_{c} / g$ and $\Delta_{2} / a$ by keeping $\omega_{1}, g, a_{1}$, and $a_{2}$ fixed and varying $\omega_{2}$ and $\omega_{c}$. At each parameter point $\left(\Delta_{c} / g, \Delta_{2} / a\right)$, there is a unique minimum duration (a quantum speed limit) for any gate operation and pre-specified error.

All parameters are listed in Table 1. We include in our model the most relevant source of dissipation, spontaneous decay of the qubits with a decay rate $\gamma$ (and the associated qubit lifetime $\tau_{\gamma}$ ), and spontaneous decay of the cavity with decay rate $\kappa$ (lifetime $\tau_{K}$ ). We assume the same decay rate for both qubits for convenience only. Different rates would result in correspondingly different error limits for single-qubit operations on each qubit, but not affect results otherwise.

$$
\begin{aligned}
& \text { Table 1. System parameters } \\
& \omega_{1} / 2 \pi=6.0 \mathrm{GHz} \\
& \omega_{2} / 2 \pi=5.0-7.0 \mathrm{GHz} \text { (vary); } \Delta 2 \equiv \omega_{2}-\omega_{1} \\
& \omega_{c} / 2 \pi=4.5-9.0 \mathrm{GHz} \text { (vary); } \Delta c \equiv \omega_{c}-\omega_{1} \\
& a_{1} / 2 \pi=-290 \mathrm{MHz} \\
& \left.\begin{array}{l}
a_{1} / 2 \pi=-290 \mathrm{MHz} \\
a_{2} / 2 \pi=-310 \mathrm{MHz}
\end{array}\right\} a \equiv\left|a_{1}+a_{2}\right| \\
& g / 2 \pi=70 \mathrm{MHz} \\
& \gamma / 2 \pi=0.012 \mathrm{MHz} ; \tau_{\gamma}=13.3 \mu \mathrm{s}^{19} \\
& \kappa / 2 \pi=0.05 \mathrm{MHz} ; \tau_{\kappa}=3.2 \mu \mathrm{s}^{57}
\end{aligned}
$$

The full equation of motion is the Liouville-von-Neumann equation with the Hamiltonian (1), and Lindblad operators $\hat{a}, \hat{b}_{1}$, and $\hat{b}_{2}$. We encode the logical subspace, denoted by $|00\rangle,|01\rangle$, $|10\rangle,|11\rangle$, as those "dressed" eigenstates of the Hamiltonian (for $\epsilon(t)=0)$ that have the largest overlap with the "bare" states $|i j n\rangle=|000\rangle,|010\rangle,|100\rangle,|110\rangle$, where $i j$, and $n$ are the quantum numbers for the first transmon, second transmon, and the cavity, respectively.

Qubit interaction, entanglement, and spontaneous decay losses for zero external drive

Creation of entanglement is typically considered the most difficult task in the implementation of a universal set of gates. The use of dressed logical states results in a static qubit-qubit interaction $\zeta$ (for $\epsilon(t)=0$ ) that may be exploited to this end. The interaction is the result of the eigenenergies $E_{00}, E_{01}, E_{10}$, and $E_{11}$ being shifted relative to the bare frame, which leads to ${ }^{2}$

$\zeta=E_{00}-E_{01}-E_{10}+E_{11}$.

The value of $\zeta$ is shown in Fig. 1 a as a function of the two parameters $\Delta_{c} / g$ and $\Delta_{2} / a$. For a horizontal and vertical slice through the parameter landscape at the point marked as $X$, Fig. $1 \mathrm{~d}-\mathrm{g}$ shows how the dressed energy levels vary with $\Delta_{c}$ and $\Delta_{2}$, and how they combine to $\zeta$. The energy shifts become especially large when any of the (bare) qubits are near-resonant with the cavity (vertical line in panels a-c at $\Delta_{c}=\omega_{c}-\omega_{1}=0$ ), or with the "anharmonic transition" (horizontal lines at $\Delta_{2} \approx \pm a$ ). They jump in sign when crossing through the resonances, see Fig. 1d, $\mathrm{f}$. The diagonal resonance line in panels $a-c$ is for $\omega_{c}=\omega_{2}$. The interaction strength can reach values $>100 \mathrm{MHz}$ when the qubit and the cavity frequencies are very close. Such a large static interaction would allow for very fast entangling gates. The fieldfree evolution for a duration $T$ induces an entangling gate with a concurrence $^{2}$

$C_{0}(\zeta, T)=\left|\sin \frac{\zeta T}{2}\right|$.

A perfectly entangling gate is first reached after $T_{\pi}=\pi / \zeta$. This time is shown in Fig. $1 \mathrm{~b}$ as a function of $\Delta_{c} / g$ and $\Delta_{2} / a$.

The spontaneous decay of the qubit with a decay rate of $\gamma$ implies a lower bound on the error of any two-qubit gate. As shown in Methods, for a fixed gate duration $T$, we find this bound to be

$\varepsilon_{\mathrm{avg}}^{0}(\gamma, T) \approx \frac{8}{10} \gamma T$.

The decay rate of the logical eigenstates may increase relative to the bare states, due to overlap with excited bare transmon or resonator levels with shorter lifetimes. The ratio of the effective ("dressed") qubit decay rate to the bare decay rate is shown in Fig. $1 c$ as a function of $\Delta_{c} / g$ and $\Delta_{2} / a$.

At the resonance lines, the increase in dissipation is most severe. However, even in the worst case, it is within only a factor of $\approx 2.3$ of the bare rate. This is in contrast to the static interaction $\zeta$ and the duration to implement an entangling gate, $T_{\pi}$, whose values span several orders of magnitude. Choosing a specific point for gate implementation within the parameter space may therefore dramatically increase implementation speed without incurring substantially larger losses. It remains to be seen, however, whether parameter space points with a large static interaction and a small $T_{\pi}$ also allow for implementation of local operations.

\section{Entanglement creation and removal}

We now consider the use of a control field $\epsilon(t) \neq 0$ for the realization of quantum gates. Before targeting specific gates that build up a universal set, we study a prerequisite-the basic capability to entangle and disentangle. To this end, we minimize 

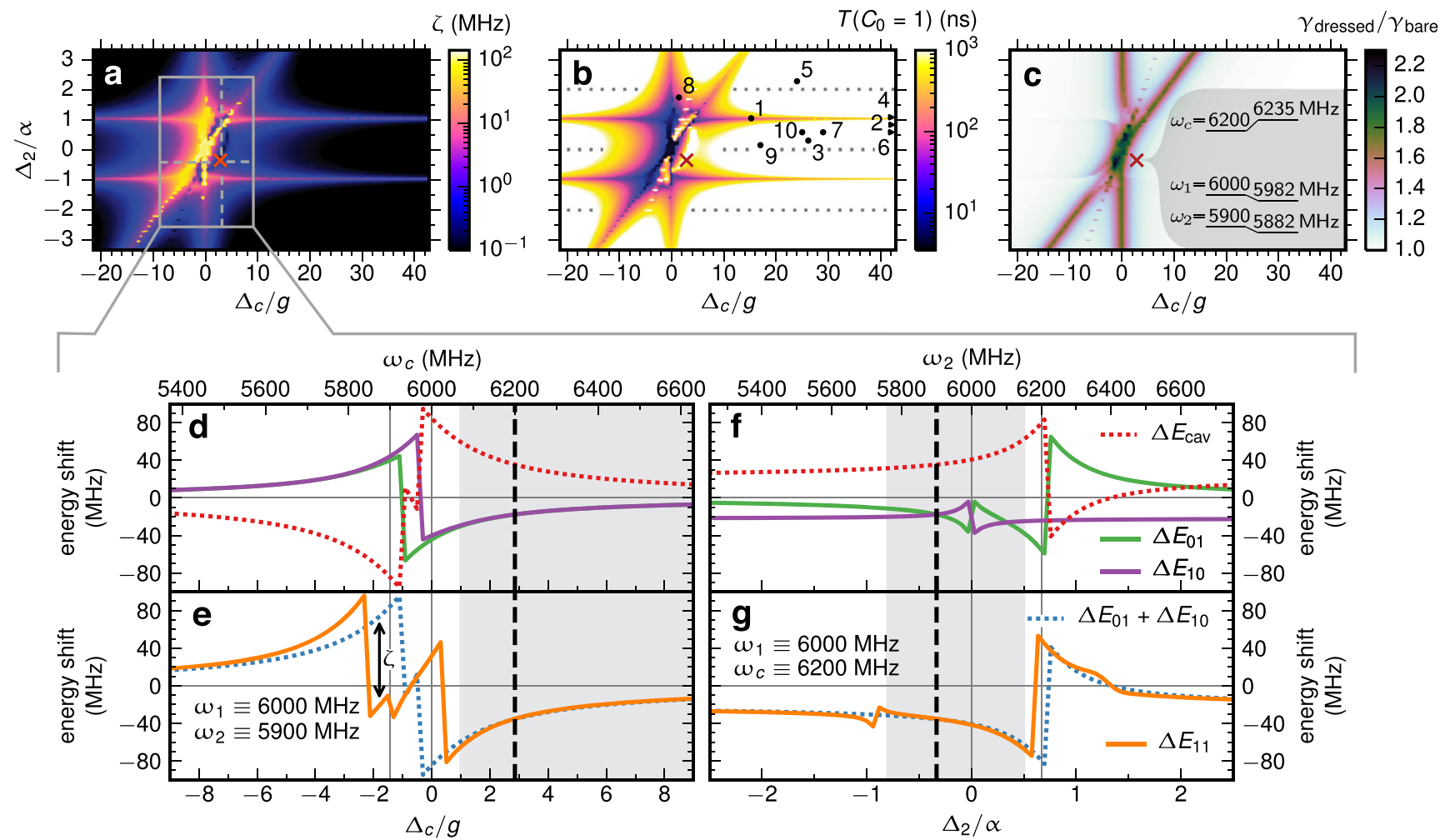

Fig. 1 Field-free properties of the parameter landscape. a Always-on interaction energy $\zeta$ resulting from the cavity-induced shift of the (dressed) qubit levels, Eq. (2). b Gate duration after which the field-free evolution produces a fully entangling gate. The points labeled 1-10 mark the parameters for some existing implementations of entangling gates for fixed-frequency transmons, cf. refs. 11-20. For points 4, 2, 6, $\Delta_{c} / g$ takes a value outside of plotted region $\left(58,95\right.$, and 43 , respectively). The gate durations are $150^{(1)}, 220^{(2)}, 110^{(3)}, 200^{(4)}, 500^{(5)}, 350^{(6)}, 350^{(7)}$, $50^{(8)}, 120^{(9)}$, and $200^{(10)} \mathrm{ns}$. The horizontal gray dotted lines indicate $\Delta_{2} / a=-2,0,2$, for visual reference. c Ratio of the dressed qubit decay rate to the bare qubit decay rate. The point labeled by the red $X$ indicates possible parameters where to implement a full universal set of gates. The bare qubit and cavity frequencies $\omega_{1}, \omega_{2}, \omega_{c}$ at this point are shown in the inset, together with their "dressed" value $E_{10}$, $E_{01}, E_{001}$, i.e., the eigenenergies of the corresponding logical levels, respectively, the eigenenergy $E_{001}$ of the eigenstate closest to the bare state $|001\rangle$. d, e For a horizontal slice through the parameter space as indicated in panel a, value of $\Delta E_{01} \equiv E_{01}-\omega_{2}, \Delta E_{10} \equiv E_{10}-\omega_{1}, \Delta E_{11} \equiv E_{11}-\omega_{1}-\omega_{2}$, and $\Delta E_{\text {cav }} \equiv$ $E_{001}-\omega_{c} . \mathbf{f}, \mathbf{g}$ Energy shifts for vertical slice as indicated in panel $\mathbf{a}$. In panels $\mathbf{e}, \mathbf{g}$ the difference between the solid orange and dotted blue curve is $\zeta$, see Eq. (2). The gray region highlights the quasi-dispersive straddling qutrits (QuaDiSQ) regime, cf. Fig. 2n. The vertical thick dashed line and thin solid lines mark the parameters of point X, and the cavity-qubit or qubit-cavity resonances, respectively

and maximize entanglement, measured by the concurrence, while minimizing loss due to decay or leakage from the logical subspace, at all points of the parameter landscape. At each point, we employ a three-stage optimization, as described in Methods.

Any entanglement that is created or removed by application of a microwave pulse is relative to the entanglement for the field-free evolution. Therefore, Fig. $2 a-c$ shows the field-free entanglement $C_{0}(\zeta, T)$ (Eq. (3)) as a function of $\Delta_{c} / g$ and $\Delta_{2} / a$ for gate durations $T=200,50$, and $10 \mathrm{~ns}$. The oscillatory behavior in Fig. $2 \mathrm{a}, \mathrm{b}$ results from the fact that $T \gg \pi / \zeta$ for these longer gate times.

The basic capability to carry out local operations, quantified as $C_{\mathrm{SQ}}$, is analyzed in Fig. $2 \mathrm{~d}-\mathrm{f}$. Entanglement can be reduced below the field-free values in a large part of the parameter space by applying suitably shaped pulses, as reflected by the dark areas in Fig. $2 d-f$. For long gate durations ( $T=200 \mathrm{~ns}$ ), the concurrence can be brought close to zero over nearly the entire parameter landscape, see Fig. 2d. As the pulse duration gets shorter, the parameter region with significant field-free entanglement becomes smaller (Fig. 2b, c). At the same time, bringing the concurrence to zero becomes more difficult. This is true in particular along the resonance lines $\omega_{c} \approx \omega_{1}, \omega_{2}$, for $-a<\Delta_{2}<a$, which is the region for which there is substantial field-free entanglement even for very short gate durations ( $T=10 \mathrm{~ns}$, Fig. 2c). Application of a pulse cannot further reduce the concurrence, see Fig. 2f. Thus, a speed limit for pulse-induced removal of the non-local nature of the interaction, $\left(C_{0}>0 \rightarrow C_{\mathrm{SQ}}=\right.$ 0 ) is found around $10 \mathrm{~ns}$.

The basic capability to create entanglement, quantified as $C_{\mathrm{PE}}$ with microwave control is shown in Fig. $2 \mathrm{~g}-\mathrm{i}$. Comparison with the field-free entanglement $C_{0}$ in Fig. $2 a-c$ reveals that adding microwave controls enlarges the region of parameter space where entanglement can be created, as expected. For long gate durations $(T=200 \mathrm{~ns}$ ), entanglement can be generated in large parts of the parameter space, in particular in the region $-a<\Delta_{2}<$ $a$ around the resonance of both qubits $\left(\Delta_{2} \approx 0\right)$. As the gate duration becomes shorter $(T=50,10 \mathrm{~ns})$, the region where perfect entanglers can be implemented shrinks (Fig. 2h, i). Still, even for very short gate durations, it is possible to generate pulse-induced perfect entanglers along the line $\Delta_{2}=0$.

Optimal quasi-dispersive straddling qutrits (QuaDiSQ) regime for implementing a universal set of gates

The realization of a full universal set of gates ${ }^{38}$ requires a region in the parameter space that allows for both entangling and local gates. This is true both for tunable and fixed-frequency qubits, since in the former case the tuning range should be kept small in order to avoid dephasing (flux) noise. To identify such regions, we inspect the product $C_{\mathrm{PE}} \times\left(1-C_{\mathrm{SO}}\right)$ in Fig. $2 \mathrm{k}-\mathrm{n}$.

It is noteworthy that points with large field-free entanglement $\zeta$, cf. Figure $1 \mathrm{a}$, are not ideal candidates for fixed-frequency qubits. This is because each qubit transition is strongly dressed by the 

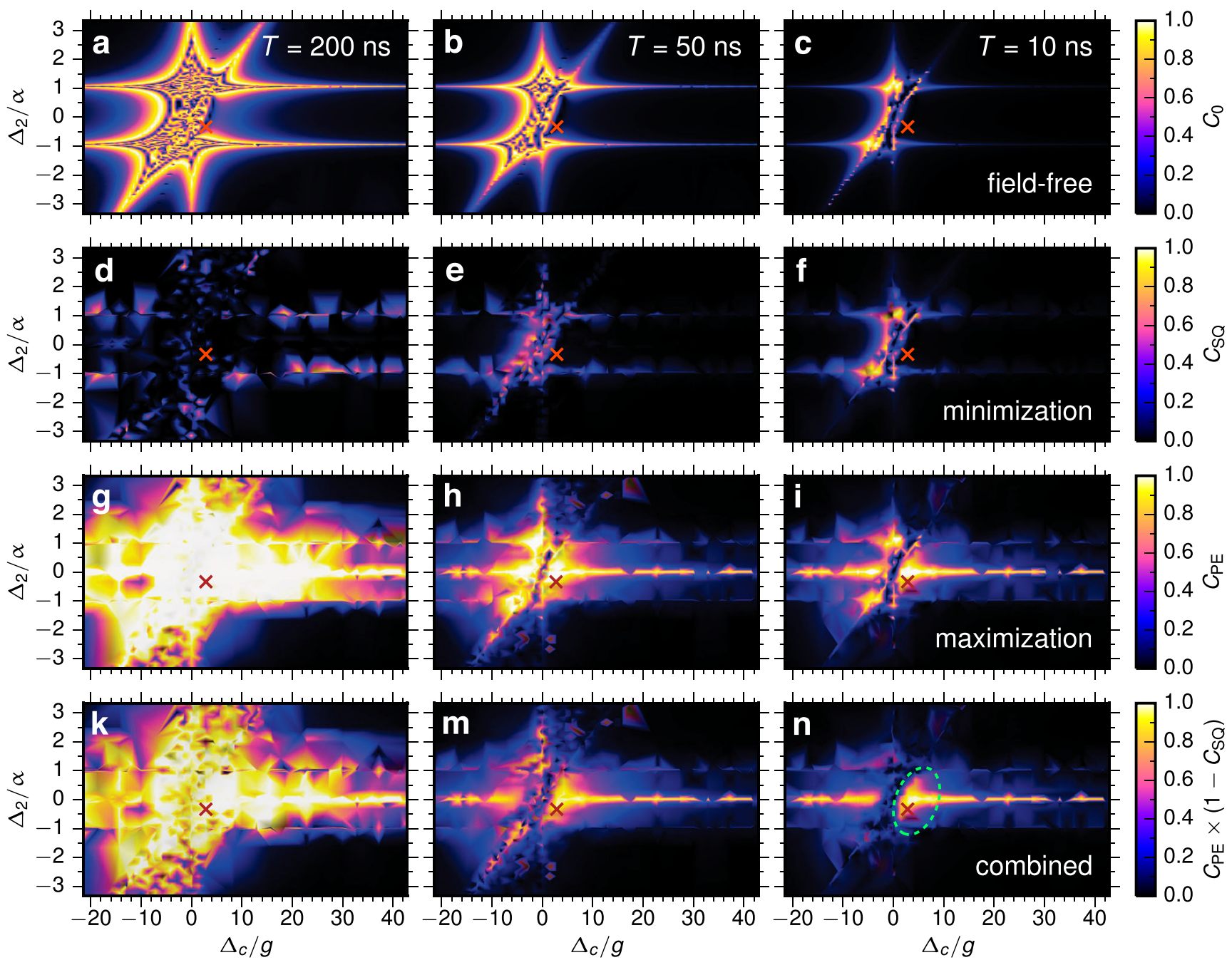

Fig. 2 Maximization and minimization of entanglement for varying gate duration. a-c Concurrence $C_{0}$ induced by the static interaction energy $\zeta$, see Fig. 1 a, after field-free evolution for $T=200,50,10 \mathrm{~ns}$. d-f Concurrence $C_{\mathrm{SQ}}$ under an optimized microwave field that minimizes entanglement. $\mathbf{g}$-i Concurrence $C_{\mathrm{PE}}$ obtained from maximization of entanglement. $\mathbf{k}-\mathbf{n}$ Combined measure of success $C_{\mathrm{PE}}\left(1-C_{\mathrm{SQ}}\right)$ for the ability to both produce local gates $\left(C_{\mathrm{SQ}}=0\right)$ and perfectly entangling gates $\left(C_{\mathrm{PE}}=1\right)$. In all panels, loss from the logical subspace (through excitation or dissipation) is indicated as transparency against the (black) background. The turquoise ellipse encircles the "QuaDiSQ" region which we identify as optimal for the control of entanglement, see text for details. The point marked by the red $\mathrm{X}$ is a candidate for the implementation of a universal set of gates

other qubit in this case. While it allows for the easy realization of an entangling (CPHASE) gate in under $10 \mathrm{~ns}^{39} \mathrm{cf}$. Figure $1 \mathrm{~b}$, it prevents single-qubit gate operations independent of the state of the other qubit. Similarly, the horizontal line in Fig. $2 m-n$ suffers from a resonance between the qubits $\left(\Delta_{2}=0\right)$, which impairs their individual addressability despite the absence of field-free entanglement.

We conclude that an optimal regime for fixed-frequency transmons requires simultaneously (1) very small static entangling strength $\zeta$ and (2) a small dispersive parameter $\left(\omega_{1,2}-\omega_{c}\right) / g$, while (3) also avoiding resonance of the qubit frequencies. This is the case in the region encircled by the turquoise dashed ellipse in Fig. 2n, for which we coin the name Quasi-Dispersive Straddling Qutrits (QuaDiSQ).

The quasi-dispersive regime of cavity-QED is defined as $1<$ $\left(\omega_{1,2}-\omega_{c}\right) / g<10$, between the near-resonant $\left(\left(\omega_{1,2}-\omega_{c}\right) / g<1\right)$ and the dispersive $\left(\left(\omega_{1,2}-\omega_{c}\right) / g>10\right)$ regime ${ }^{40}$, both of which are excluded by conditions (1)-(3). Meanwhile, the minimal static coupling $\zeta$ between the qubits ( $c f$. black/white disc in Fig. 1a, b, and crossing solid orange and dotted blue lines in Fig. 1e, f) is enabled by one transmon frequency being situated between the first and second $(|1\rangle \rightarrow|2\rangle)$ transition of the other transmon (hence "qutrits"), $-1<\Delta_{2} / a<1$. This region is reminiscent of the so-called straddling regime of single-transmon circuit $\mathrm{QED},{ }^{9}$ with the distinction that the qutrits are straddling one another here, rather than the cavity. This allows the level repulsion to act with opposite signs, see Fig. $1 \mathrm{~d}, \mathrm{f}$, effectively resulting in destructive interference and avoiding unwanted entanglement. The qubits being sufficiently separated still allows individual addressing with microwave pulses. In particular, frequency crowding is also avoided, with the nearest unwanted transition at least $100 \mathrm{MHz}$ detuned for all the relevant transitions. We emphasize that this optimized mechanism is a general principle for quantum information processing, whereby destructive interference can be engineered on a multi-qubit state to mutually cancel out level repulsions coming from nearby levels and thereby enable both driving of local and nonlocal transitions from the level.

Lastly, the decoherence rate is only marginally increased in the quasi-dispersive regime relative to the bare rate, cf. Fig. 1c. At the point marked $X$ in Figs. 1 and 2 the corresponding error limit is shown in Fig. $4 \mathrm{~b}$ (blue dashed vs. solid gray line). Thus, point $X$ in Figs. 1 and 2 provides an effective optimum for these combined 
error mechanisms. The exact corresponding parameters are listed in Fig. 1c.

Quantum speed limit for a universal set of gates

A universal set requires one entangling gate, with many choices available. We, therefore, analyze which perfect entanglers can be implemented before determining the quantum speed limit for a universal set of gates. To this end, we employ the representation of two-qubit gates in the Weyl chamber (see Methods). Figure 3 indicates the perfect entanglers that are successfully implemented in Fig. $2 g-i$. For long gate durations ( $T=200 \mathrm{~ns}$, Fig. 3a), a large part of the polyhedron of perfect entanglers is covered. That is, nearly any perfect entangler can easily be implemented. This changes as the pulse duration gets shorter. For $T=50 \mathrm{~ns}$ (Fig. 3b), the reached perfect entanglers are focused around the $L$ point (diagonal gates), and the lines $\mathrm{Q}-\mathrm{A}_{2}$ and $\mathrm{Q}-\mathrm{P}, \mathrm{M}-\mathrm{N}$ (local equivalence classes of $\sqrt{\mathrm{iSWAP}}-\sqrt{\mathrm{SWAP}}$ ). We can, therefore, empirically identify the dominant effective qubit-qubit interaction term as a linear combination of $\hat{\sigma}_{x} \hat{\sigma}_{x}, \hat{\sigma}_{y} \hat{\sigma}_{y}$, and $\hat{\sigma}_{z} \hat{\sigma}_{z}$. For very short gate durations ( $T=10 \mathrm{~ns}$, Fig. $3 \mathrm{c}$ ), the obtained gates cluster strongly around the points $\mathrm{Q}$ and $\mathrm{M}$ (local equivalence class of $\sqrt{\text { iSWAP) }}$, corresponding to a dominant interaction term $\hat{\sigma}_{x} \hat{\sigma}_{x}+\hat{\sigma}_{y} \hat{\sigma}_{y}=\hat{\sigma}_{+} \hat{\sigma}_{-}+\hat{\sigma}_{-} \hat{\sigma}_{+}$. This is in agreement with the interaction obtained from effective two-qubit models in the dispersive regime. ${ }^{41}$ Our results suggest that targeting $\sqrt{\text { iSWAP }}$ (or a gate that is locally equivalent to $\sqrt{\mathrm{iSWAP}}$ ) is natural also in the non-dispersive case.

Analysis of the gate fidelities of the perfect entanglers allow us to extract the quantum speed limit for the generation of entanglement. The smallest error of any perfect entangler in Fig. $2 \mathrm{~g}-\mathrm{i}$ is shown as $\varepsilon_{\text {avg }}^{\mathrm{PE}}$ in Fig. $4 \mathrm{a}$, together with results for additional gate durations. $\varepsilon_{\mathrm{avg}}^{\mathrm{PE}}$ is to be compared to the error $\varepsilon_{\mathrm{avg}}^{0}$ due to qubit decay, Eq. (4). For $T>10 \mathrm{~ns}$, we find the errors to be limited by the qubit lifetime. For $T=5 \mathrm{~ns}$, the error increases significantly from the lifetime-limited bound, indicating that the main source of error is now the lack of capability to create entanglement. We thus find $T \approx 10 \mathrm{~ns}$ to be the minimum time necessary to build entanglement.

Next, we determine the quantum speed limit for local gates in the QuaDiSQ regime, using the parameters of point $X$. The smallest gate error of a Hadamard gate on qubit $1(\mathrm{H} \otimes 1)$ is shown as $\varepsilon_{\mathrm{avg}}^{\mathrm{H} 1 \mathrm{X}}$ in Fig. $4 \mathrm{~b}$. We find that the Hadamard gate can be implemented near a lifetime-limited error bound up to a gate duration of $50 \mathrm{~ns}$. Targeting the remaining single-qubit gates in the universal set, $1 \otimes \mathrm{H}, S_{\pi / 8} \otimes 1$, and $1 \otimes S_{\pi / 8}$ (with $S_{\pi / 8}=\operatorname{diag}[1$, exp $(-i \pi / 4)])$ yields comparable errors. We, therefore, identify $50 \mathrm{~ns}$ as the speed limit for single-qubit operations.

For implementing a general perfect entangler at point $\mathrm{X}$ with a gate error close to the theoretical limit, we find a minimum gate duration of $T=20 \mathrm{~ns}$, located in the Weyl chamber on the line Q-P. This is slightly larger than the global limit of $10 \mathrm{~ns}$. It is worth noting that this entanglement rate is nonetheless much faster than what is achievable through resonant qubit-coupling (typically limited to the inverse of the cavity-mediated qubit-qubit coupling, $\approx 20 \mathrm{MHz}$ at point $\mathrm{X}$ ). This suggests that even for a frequencytunable architecture, it can be advantageous to supplement static qubit coupling with microwave drives for even faster gates.

When decomposing an arbitrary computation of a quantum computer into a universal set of single and two-qubit gates, it is important to use an entangling operation in this set that yields the smallest possible number of elementary gates. To this end, the perfect entangler must be taken from a small subset of "special" perfect entanglers. Specifically, gates along the line $L-A_{2}$ in the Weyl chamber are known to be efficient. ${ }^{42}$ The mid-point of this line, known as the BGATE (see Methods), allows for the most efficient realization of a universal set. It requires at most two applications in the decomposition of an arbitrary gate, compared to three for iSWAP. We, therefore, target a BGATE at parameter point X. Compared to iSWAP/ $\sqrt{\text { iSWAP, this entails small tradeoffs }}$ in the gate duration and in the complexity of the control pulses. We find a minimum gate duration for a BGATE of $50 \mathrm{~ns}$.

Realization of a universal set of gates

The optimized pulses and the corresponding dynamics (in the interaction picture) for the entire universal set of gates, consisting of $\mathrm{H} \otimes 1,1 \otimes H, S_{\pi / 8} \otimes 1,1 \otimes S_{\pi / 8}$, and BGATE, are shown in Fig. 5. The average gate error over the entire universal set is $4.3 \times 10^{-3}$ compared to the lifetime-limited error bound of $\varepsilon_{\text {avg }}^{0}=3.6 \times 10^{-3}$.

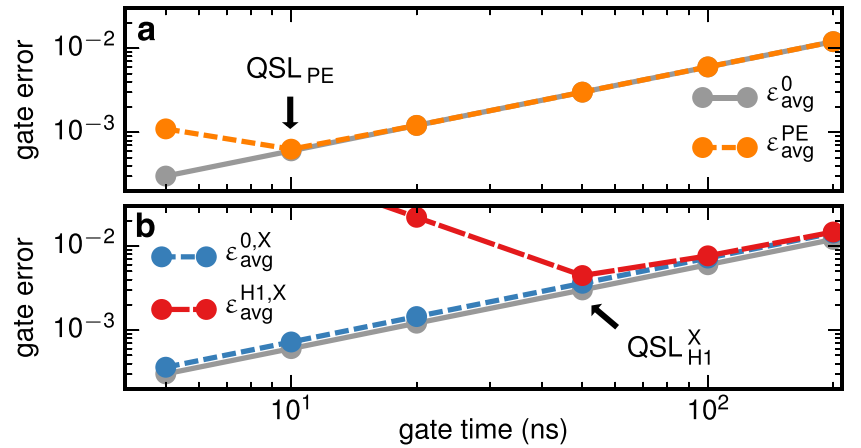

Fig. 4 Quantum speed limit (QSL) for the generation of a perfect entangler, and a local quantum gate. a Minimum gate error $\varepsilon_{\mathrm{avg}}^{\mathrm{PE}}$ of perfect entanglers over the entire parameter landscape, compared to the lifetime-limited error bound $\varepsilon_{\text {avg }}^{0}$ defined in Eq. (4). The minimum in $\varepsilon_{\mathrm{avg}}^{\mathrm{PE}}$ represents the quantum speed limit for the implementation of a perfect entangler. $\mathbf{b}$ Gate error $\varepsilon_{\mathrm{avg}}^{\mathrm{H1}, \mathrm{X}}$ of a Hadamard gate on qubit 1 at point $X$ (Figs. 1 and 2 ) in the optimal QuaDiSQ regime, compared to the lifetime-limited error bound, $\varepsilon_{\text {avg }}^{0, X}$ at point $X$, which differs from the global limit $\varepsilon_{\text {avg }}^{0}$ by a factor of 1.2 due to the increase in the effective decay rate, cf. Fig. 1c. The minimum in $\varepsilon_{\mathrm{avg}}^{\mathrm{H1}, \mathrm{X}}$ may be taken as an estimate of the global quantum speed limit for implementing a universal set of gates
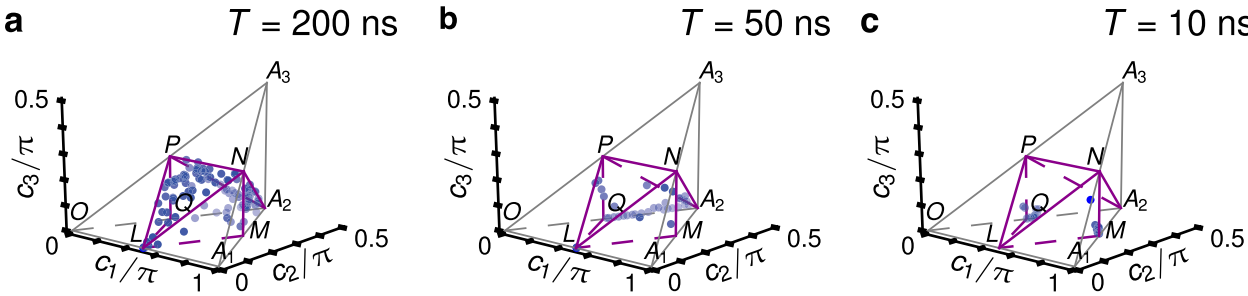

Fig. 3 Location in the Weyl chamber of all perfect entanglers (blue dots, lighter shades farther back) reached by the maximization of entanglement in Fig. 2g-i. The total Weyl chamber and the polyhedron of perfect entanglers are indicated by thin gray and bold magenta lines, respectively 


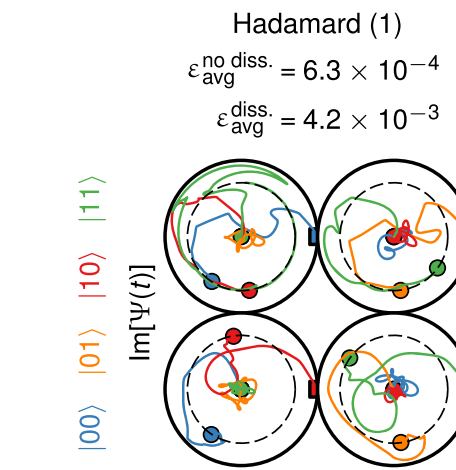

$\operatorname{Re}[\Psi(t)]$

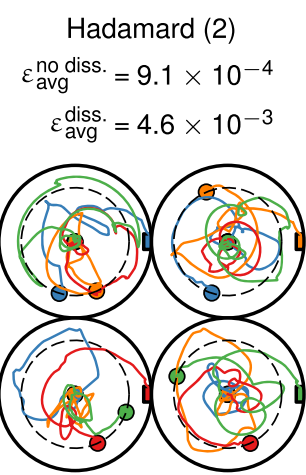

$\operatorname{Re}[\Psi(t)]$

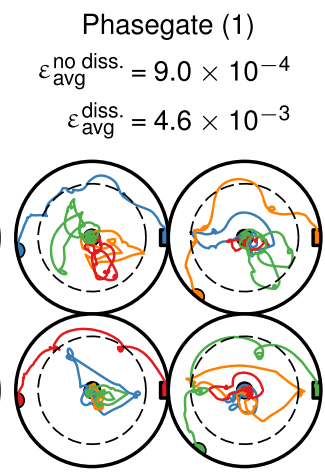

$\operatorname{Re}[\Psi(t)]$

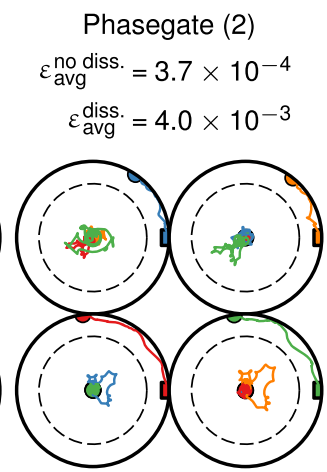

$\operatorname{Re}[\Psi(t)]$
BGATE

$\varepsilon_{\mathrm{avg}}^{\text {no diss. }}=6.5 \times 10^{-4}$

$\varepsilon_{\mathrm{avg}}^{\text {diss. }}=4.3 \times 10^{-3}$

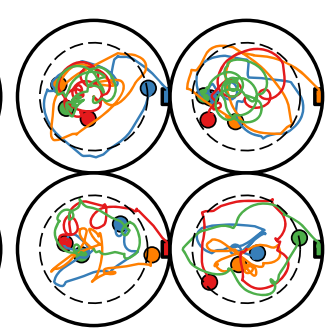

$\operatorname{Re}[\Psi(t)]$
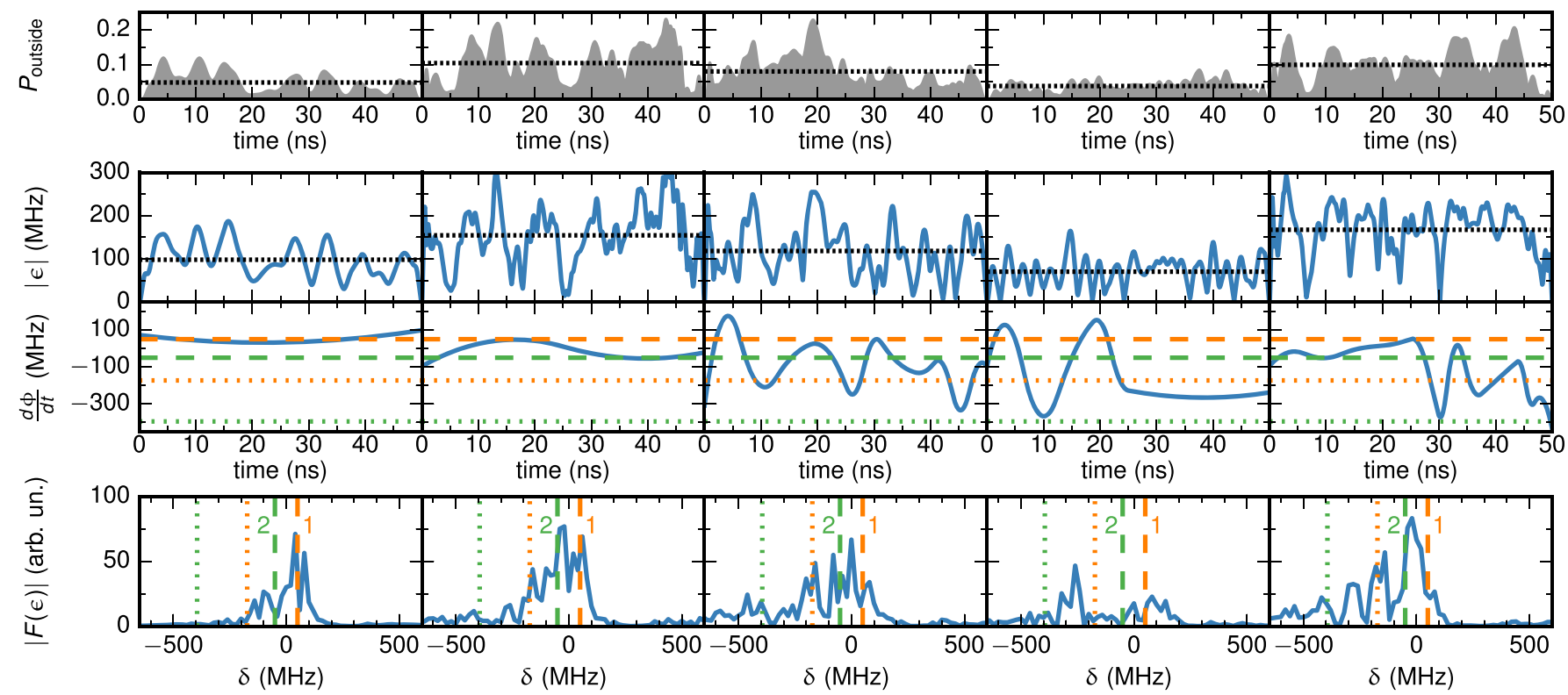

Fig. 5 Optimized pulses and dynamics implementing a universal set of gates (Hadamard and phase gate $S_{\pi / 8}$ on both qubits, and non-local

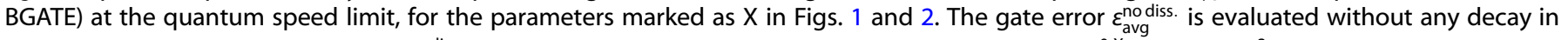
the system. The dissipative gate error $\varepsilon_{\text {avg }}^{\text {diss. }}$ should be compared to the lifetime-limited error bound $\varepsilon_{\text {avg }}^{0, x}=3.6 \times 10^{-3}$ for an assumed lifetime of $13.3 \mu \mathrm{s}$. The dynamics are shown for each of the four logical basis states, in terms of amplitude and phase of the projection onto the logical basis states (color-coded) within the unit circle. The dashed circle marks an amplitude of $1 / \sqrt{2}$. The values at initial (final) time are indicated by the colored squares (bullets). The population $P_{\text {outside }}$ outside of the logical subspace is plotted over time. The properties of the optimized pulse $\epsilon(t)$ for each gate are shown, from top to bottom, in terms of the pulse amplitude $|\epsilon|$, the (smoothed) derivative of the complex phase $\mathrm{d} \phi / \mathrm{d} t$, and the spectrum $|F(\epsilon(t))|$ for frequencies $\delta$ relative to the rotating frame at $\omega_{r} / 2 \pi=5.9325 \mathrm{GHz}$. The derivative $\mathrm{d} \phi / \mathrm{d} t$ gives an approximation for $\delta(t)$. The dressed qubit frequencies are indicated as green and orange dashed lines, the dressed "anharmonic transitions" $|1\rangle \rightarrow|2\rangle$ as green and orange dotted lines. The black dotted lines correspond to the mean value of $P_{\text {outside }}$ and $|\epsilon|$, respectively

In all cases, the achieved gate error is within a factor of 1.3 of the lifetime-limited error bound. It is correlated with the mean of the population outside the logical subspace, $P_{\text {outside, }}$ which in turn is correlated with the mean pulse amplitude (black dotted horizontal lines). Population that is excited to these higher levels is more strongly affected by dissipation, because the decay rate scales as the square root of the (bare) quantum numbers. In fact, evaluating

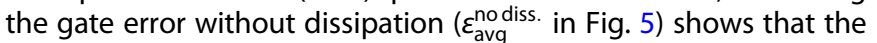
error is dominated by the decay of the qubit, not by failure to implement the desired gate. As qubit lifetimes increase with further technological advances of the transmon platform, errors will approach the value $\varepsilon_{\text {avg }}^{\text {no diss. }}$, consistently below the quantum error correction limit.

For all gates, we obtained the lowest error when the optimization was performed in a rotating frame with frequency centered exactly between the two qubit frequencies. While for the implementation of the Hadamard gate, the spectra show active frequencies largely around the two qubit transitions (indicated by the dashed green and orange lines), for the phase gate as well as the BGATE, the pulses also have strong off-resonant components. These are predominantly to the left of the qubit transitions, and thus affect the anharmonic transitions more strongly, driving pathways outside of the logical subspace.

The derivative of the complex phase, $\mathrm{d} \phi / \mathrm{d} t$ in Fig. 5 provides a rough estimate of the time-frequency characteristics of the optimized pulse. For several of the pulses, we find distinct differences in the active frequencies over the duration of the pulse. For example, for $1 \otimes H$, the pulse alternates between the two qubit frequencies. For $1 \otimes S_{\pi / 8}$, the strongly off-resonant driving is interrupted by two brief periods of near-resonant driving. For the BGATE, the first half of the pulse acts near-resonant on the two qubits, whereas in the second half, strong off-resonant kicks are applied. It will be interesting to see in future work whether the observed feature of alternating periods of near-resonant and offresonant driving may be exploited in an analytic design of control fields near the quantum speed limit.

In practice, any waveform generator will also have bandwidth and filtering restrictions that must be taken into account. ${ }^{37,43,44}$ 
In order to identify the quantum speed limit, we have not considered any such restrictions. Consequently, the pulses have bandwidths in the $500-1000 \mathrm{MHz}$ range. While this is well within reach of the most current waveform generators, ${ }^{45}$ it may be beyond the limitations of older devices typically used in experiments. ${ }^{43}$ In such a case, moving away from the speed limit of $T=50 \mathrm{~ns}$ to e.g., $T=100 \mathrm{~ns}$ may provide several practical advantages, as the longer gate durations give room for applying spectral constraints. Furthermore, gate errors will approach the lifetime limit more closely, giving some room to compensate for imperfections in the control scheme. In the Supplementary Material, we show pulses implementing a universal set of gates at $T=100 \mathrm{~ns}$ with a spectral constraint of $\pm 200 \mathrm{MHz}$ around the center of the rotating frame.

Robustness with respect to fluctuations can also be added as an explicit optimization objective. ${ }^{46}$ For example, for a Hadamard gate on transmon 1 at a longer gate duration of $T=100 \mathrm{~ns}$, we find that by simultaneously optimizing over multiple variations of the system we can account for errors in the pulse amplitude of $1 \%$ with relative ease, incurring a worst-case drop in the gate error from $7.3 \times 10^{-3}$ to $7.6 \times 10^{-3}$, or $8.9 \times 10^{-5}$ to $3.7 \times 10^{-4}$ without spontaneous decay of the qubit. In general, both technical constraints and noise sources should be addressed with respect to a specific experimental set-up. Optimal control techniques toward this end are readily available. ${ }^{47,48}$

\section{DISCUSSION}

Superconducting qubits with a shared transmission line come with great tunability in system parameters. We have addressed the question of how to choose these parameters in order to implement a universal set of gates with the best possible errors and shortest possible gate durations. We have found that the parameter landscape may be fully characterized by the qubitqubit detuning in units of the anharmonicity and the qubit-cavity detuning in units of the qubit-cavity coupling. Analysis of the fieldfree qubit dynamics revealed novel strategies for implementing both entangling and local quantum gates. Resonances between qubit levels, or with the cavity, can generate very large interactions even without any external drive. In these regions of the parameter space, we showed that a quantum speed limit of 10 ns can be obtained for the controlled generation or removal of entanglement. However, a strong, always-on interaction cannot yield a fast universal set of quantum gates with low error, due to the difficulty to generate specific single qubit gates. These regions of the parameter space are, therefore, only of interest to setups that employ tunable qubits. For fixed-frequency qubits, highfidelity quantum gates are best implemented with system parameters that do not yield any static interaction but where strong interaction can be engineered in a time-dependent fashion by a suitably shaped microwave pulse.

A key result of this work is the demonstration that the conditions for the realization of a universal set of gate for short gate durations are best met outside of the dispersive regime. Parameter regimes where the dispersive approximation is not valid have remained under-explored to date, since the Hamiltonian in this regime cannot easily be approximated to an analytically treatable model. In this work, we have used a fully numerical approach to explore the complete parameter space without restrictions and thereby identified a novel parameter regime as the optimal operating regime for universal quantum computing in which the global quantum speed limits for a given architecture are attained. In this new regime, which we term the Quasi-Dispersive Straddling Qutrits (QuaDiSQ) regime, we have shown that two critical but competing requirements for realizing a universal set of gates are achieved through destructive interference of multiple near-resonances. Specifically, one can maintain the near-resonances that allow for fast entangling gates, while also minimizing static qubit-qubit interaction allowing for the implementation of local gates.

Using a universal set of gates consisting of the non-local BGATE, as well as Hadamard and phase gates on each of the two qubits, we derived control protocols to realize gates with errors within a factor of 1.3 of the lifetime-limited error bound for a gate duration of 50 ns. Given this identification of the new QuaDiSQ regime and characterization of the fundamental quantum speed limits, further requirements and constraints of a specific experimental set-up can be taken into account. For example, by extending the gate duration to $100 \mathrm{~ns}$, we can enforce a spectral width of the pulses of $\pm 200 \mathrm{MHz}$ around the center frequency. Robustness to experimental parameters such as variations in the pulse amplitude can further be taken into account for specific setups.

Other choices for the entangling operation are conceivable as well. For long gate durations, almost all perfect entanglers can be implemented. When the gate duration is shortened, only the $\sqrt{\text { iSWAP }}$ gate plus all gates that differ from $\sqrt{\text { iSWAP }}$ by local operations survive. This indicates that $\sqrt{\text { iSWAP }}$ is the most natural entangling gate for the transmon architecture. This is in agreement with earlier findings obtained in the dispersive regime, ${ }^{41}$ suggesting that effective two-qubit models may be still be qualitatively correct even when they break down quantitatively.

The approach advocated here of identifying a few key parameters and exploring those with QOC is not limited to circuit QED platforms nor quantum computation. In fact, the paradigm of quantum technology is to engineer an often complex quantum system to resemble a comparatively simple model Hamiltonian (an anharmonic ladder system in the case of the transmon). Quantum engineering ensures, on one hand, that only a few parameters will be relevant in the analysis of the possible dynamics. On the other hand, isolation of the required quantum features typically implies limited control over the system. Ensuring the desired functionality (here a universal set of quantum gates) despite limited control (no or limited tunability of the qubits in the present case) is possible by harnessing some of the complexity of the quantum system (using multiple interfering sources of entanglement, as in the QuaDiSQ regime in our example). This paradigm finds many realizations at the interface of quantum optics and solid-state physics, for the design of devices to tackle tasks such as sensing of external fields with quantum-enhanced sensitivity or transmission of quantum information with cryptographic capability. QOC is essential to achieving this goal.

\section{METHODS}

\section{Optimization procedure}

The results shown in Fig. 2 are the result of optimal control theory applied to a large sample of parameter points $\left(\Delta_{2} / a, \Delta_{c} / g\right)$. At each point, a multistage optimization procedure ${ }^{49}$ is employed to either minimize or maximize entanglement, proceeding in three stages.

In the first stage, we presume the control pulse to take the form

$\epsilon(t)=E_{0} B(t) \cos \left(\omega_{r} t\right) \stackrel{\mathrm{RWA}}{\longrightarrow} E_{0} B(t)$,

where $B(t)$ is a Blackman shape, similar to a Gaussian, but exactly zero at initial and final time. In the rotating frame, the driving frequency $\omega_{r}$ vanishes and instead is reflected in the Hamiltonian (1). The width of the shape is constant, extending over the entire duration. Thus, the control problem has only two free parameters, the peak amplitude $E_{0}$ and the driving frequency $\omega_{r}$. Note that a complex $\epsilon(t)$ would be equivalent to a time-dependent phase of the pulse in the non-rotating frame. However, we find that the results in Fig. 2 do not change significantly when allowing for multiple frequency components or allowing a non-zero phase.

The first optimization stage consists in choosing $\omega_{r}$ randomly from within a range of $1 \mathrm{GHz}$ around the qubit and cavity frequencies, and scanning the pulse amplitude $E_{0}$ systematically for values $\in[10,900] \mathrm{MHz}$. 
The best pulses are selected according to the functionals

$J_{\mathrm{PE}}^{\mathrm{splx}}=1-C\left(1-\varepsilon_{\mathrm{pop}}^{\mathrm{min}}\right)$,

$J_{\mathrm{SQ}}^{\mathrm{splx}}=1-(1-C)\left(1-\varepsilon_{\mathrm{pop}}^{\mathrm{min}}\right)$.

This takes into account both the concurrence $C$ and the error due to population loss,

$\varepsilon_{\text {pop }}^{\min }=1-\min _{i} \| \hat{\mathrm{U}}|i\rangle \| ; \quad i \in\{|00\rangle,|01\rangle,|10\rangle,|11\rangle\}$,

where $\hat{U}$ is the projection of the time evolution operator onto the logical subspace. The functional is written as a product, since the concurrence is only well-defined for a population loss error near zero.

The selected pulses are the starting point for the second stage, a simplex (Nelder-Mead) optimization of the two free parameters $E_{0}$ and $\omega_{r}$ in Eq. (5), using the same optimization functionals (6), (7). The third and last stage of optimization relaxes the constraint imposed by the simple analytical form (5), and uses Krotov's method ${ }^{50}$ to continue optimization of $\epsilon(t)$ for an arbitrary perfect entangler, respectively, an arbitrary local gate. As any gradient-based optimization approach, Krotov's method requires a differentiable functional. Since the concurrence is non-analytic in the control, we cannot employ Eqs. (6) and (7) and need to resort to optimization in the Weyl chamber as described below. The pulse shape is now allowed to take complex values. The total optimization functional also includes a term $\operatorname{tr}\left[\hat{U}^{\dagger} \hat{U}\right] / 4$ to penalize loss from the logical subspace. ${ }^{51}$

The optimization for a complete universal set of gates uses a similar three-stage procedure as outlined above, but optimizes toward the BGATE (up to local operations, see below) and toward the four local gates using the gate overlap with the target gate $\hat{O}$, $F_{\mathrm{sm}}=\left|\operatorname{tr}\left[\hat{\mathrm{O}}^{\dagger} \hat{\mathrm{U}}\right]\right|^{2} ; \quad J_{\mathrm{sm}}=1-F_{\mathrm{sm}}$.

Optimization in the Weyl chamber

Any two-qubit gate $\hat{U} \in \mathrm{SU}(4)$ can be written according to the Cartan decomposition, 52,53

$\hat{\mathrm{U}}=\hat{\mathrm{k}}_{1} \exp \left[\frac{i}{2}\left(c_{1} \hat{\sigma}_{x} \hat{\sigma}_{x}+c_{2} \hat{\sigma}_{y} \hat{\sigma}_{y}+c_{3} \hat{\sigma}_{z} \hat{\sigma}_{z}\right)\right] \hat{\mathrm{k}}_{2}$,

where $\hat{\sigma}_{x}, \hat{\sigma}_{y}, \hat{\sigma}_{z}$ are the Pauli matrices, and $\hat{\mathrm{k}}_{1,2} \in \mathrm{SU}(2) \otimes \mathrm{SU}(2)$ are singlequbit, "local" operations. Taking into account symmetries, the coefficients $c_{1}, c_{2}, c_{3}$ take values $c_{1} \in[0, \pi]$ and $c_{2}, c_{3} \in[0, \pi / 2]$. They may be interpreted as coordinates in a three-dimensional space; all the points that represent the possible two-qubit gates up to local operations then form a quarterpyramid called the Weyl-chamber. It is depicted in Fig. 3 . The named edges and vertex midpoints of the Weyl chamber correspond to some of the "standard" two-qubit gates (CNOT, CPHASE, SWAP, iSWAP, etc.). The point $(\pi / 2, \pi / 4,0)$, i.e., the midpoint of the line $L-A_{2}$, defines the BGATE that is the optimal perfect entangler for the universal set of gates, with the canonical representation

$\mathrm{BGATE}=\left(\begin{array}{cccc}\cos \frac{\pi}{8} & 0 & 0 & i \sin \frac{\pi}{8} \\ 0 & \cos \frac{3 \pi}{8} & i \sin \frac{3 \pi}{8} & 0 \\ 0 & i \sin \frac{3 \pi}{8} & \cos \frac{3 \pi}{8} & 0 \\ i \sin \frac{\pi}{8} & 0 & 0 & \cos \frac{\pi}{8}\end{array}\right)$.

Separating a quantum gate into local and non-local contributions through the Cartan decomposition allows to optimize for a quantum gate up to local operations by minimizing the "local invariants functional" $J_{L l}$. It evaluates the geometric distance to a target point in the Weyl chamber. ${ }^{51}$ This is especially appropriate in the context of universal quantum gates, as we assume that arbitrary single-qubit gates can be implemented.

The majority of two-qubit entanglers which form a polyhedron with the edge points $L, M, A_{2}, Q, P$, and $N$, indicated in magenta in Fig. 3. In order to optimize for an arbitrary perfect entangler using a gradient-based method, one can define a "perfect entanglers functional" $J_{P E}$ that minimizes the geometric distance to the surface of the polyhedron ${ }^{54}$. Note that the opposite, optimizing for an arbitrary local gate (non-entangling), is achieved by applying the local invariants functional toward the points $O$ or $A_{1}$. Only when we need to implement a specific gate (e.g., the singlequbit gates in the universal set) do we employ the gate overlap $J_{\mathrm{sm}}$.

Any of the functionals defined in the Weyl chamber assume that $\hat{U} \in \mathrm{SU}(4)$. When the model includes levels outside the logical subspace, as is the case here, some of the population may remain in those spurious levels at final time $T$. The resulting projection $\widetilde{U}$ to the logical subspace may no longer be unitary. In this case, we must include the loss in the functional, ${ }^{51}$ and evaluate $J_{\mathrm{LI}}$ or $J_{\mathrm{PE}}$ using the closest unitary gate $\hat{U}$. If $\widetilde{U}$ has the singular value decomposition $\widetilde{U}=\hat{V} \hat{\Sigma} \hat{W}^{\dagger}$, then the closest unitary is

$$
\hat{U}=\arg \min _{\hat{U}}\left\|\widetilde{U}-\hat{U}^{\prime}\right\|=\hat{V} \hat{W}^{\dagger} \text {. }
$$

When evaluating the optimization success using $J_{L l}$, we must find the local operations $\hat{\mathrm{k}}_{1}, \hat{\mathrm{k}}_{2}$ in the Cartan decomposition such that $\varepsilon_{\text {avg }}$ is minimized. This is done by parametrizing an arbitrary single-qubit gate as

$\hat{\mathrm{U}}_{1 q}=e^{i \phi}\left(\begin{array}{cc}\cos \theta e^{i \phi_{1}} & \sin \theta e^{i \phi_{2}} \\ -\sin \theta e^{-i \phi_{2}} & \cos \theta e^{-i \phi_{1}}\end{array}\right)$.

With $\hat{\mathrm{k}}_{1}$ and $\hat{\mathrm{k}}_{2}$ consisting of two single-qubit gates each, this gives a total of 16 free parameters that are easily determined through numerical minimization. For $J_{\mathrm{PE}}$, the procedure is the same, except that the appropriate value of the Weyl coordinates $c_{1}, c_{2}, c_{3}$ must first be determined by projecting $\hat{U}$ onto the surface of the polyhedron of perfect entanglers (assuming $\hat{U}$ itself is not already a perfect entangler).

\section{Average gate error}

While the functionals $J_{\mathrm{L}}, J_{\mathrm{PE}}$, or $J_{\mathrm{sm}}$ are suitable for numerical purposes to steer an optimization, they are not directly accessible to measurement and can thus not be used to objectively evaluate the success of gate implementation. The experimentally relevant measure of success for implementing the target gate $\hat{O}$ with a dynamical map $\mathcal{E}$ is the average gate fidelity

$F_{\text {avg }}=\int\left\langle\Psi\left|\hat{O}^{\dagger} \mathcal{E}(T, 0)[|\Psi\rangle\langle\Psi|] \hat{\mathrm{O}}\right| \Psi\right\rangle d \Psi$,

respectively, the average gate error $\varepsilon_{\text {avg }}=1-F_{\text {avg }}$. The error is easily evaluated numerically. ${ }^{55}$ We report all gate errors in terms of $\varepsilon_{\text {avg' }}$ independent of the functional used in the optimization.

In order to derive a lower limit for the achievable gate error for a given qubit decay rate, i.e., the lifetime-limited error bound, we consider the Hamiltonian of Eq. (1) with $g=0$ and $\epsilon(t) \equiv 0$. The cavity can then be integrated out, and the transmon Hilbert space can be truncated to two levels. In the interaction picture and without dissipation, there is no time evolution $(\hat{\mathrm{O}}=1)$. The Liouville-von-Neumann equation can then be solved analytically for the qubit decay rate $\gamma$. Plugging the result into the formula for $\varepsilon_{\text {avg }}$ yields

$\varepsilon_{\text {avg }}^{0}(\gamma, T)=\frac{3}{4}-\frac{3 e^{-\gamma T}}{10}-\frac{e^{-2 \gamma T}}{20}-\frac{e^{-\frac{\gamma T}{2}}}{5}-\frac{e^{-\frac{3 \psi}{2} T}}{5}$.

For $\gamma T \ll 1$, this can be linearized to Eq. (4).

\section{Equation of motion}

In order to evaluate the average gate error for any of the optimized pulses, we numerically solve the full Liouville-von-Neumann equation

$\frac{\partial}{\partial t} \hat{\rho}=-i[\hat{\mathrm{H}}, \hat{\rho}]+\sum_{i=1}^{3}\left(\hat{\mathrm{A}}_{i} \hat{\rho} \hat{\mathrm{A}}_{i}^{\dagger}-\frac{1}{2}\left\{\hat{\mathrm{A}}_{i}^{\dagger} \hat{\mathrm{A}}_{i}, \hat{\rho}\right\}\right)$,

with the Hamiltonian (1), and the Lindblad operators $\hat{A}_{1}=\sqrt{\gamma} \hat{b}_{1}$, $\hat{\mathrm{A}}_{2}=\sqrt{\gamma} \hat{\mathrm{b}}_{2}$, and $\hat{\mathrm{A}}_{3}=\sqrt{\kappa} \hat{\mathrm{a}}$. The Hilbert space of the two transmons and the cavity are truncated at 5 and 6 levels, respectively. This has been checked to be sufficient for all pulses considered here.

For the purpose of optimization, solving Eq. (8) is numerically too expensive. Instead, we solve the Schrödinger equation with an nonHermitian effective Hamiltonian to mimic population loss,

$\hat{\mathrm{H}}_{\mathrm{eff}}=\hat{\mathrm{H}}-\frac{i \hbar}{2} \sum_{i} \hat{\mathrm{A}}_{i}^{\dagger} \hat{\mathrm{A}}_{i}$.

We also increase the decay rate of the highest qubit and cavity level to infinity as a method to ensure that the optimized pulses are well-described in the truncated Hilbert space. We stress that this non-Hermitian Hamiltonian is only used to effectively penalize population in strongly dissipative levels during the optimization. The final optimized pulses are then evaluated by solving Eq. (8); all reported dynamics and errors are obtained from this density matrix evolution. Both the Liouville-vonNeumann equation and the Schrödinger equation with a non-Hermitian 
Hamiltonian can be solved efficiently and to high precision using an expansion into Newton polynomials in a Krylov subspace, ${ }^{56}$ as implemented in the Fortran QDYN package.

\section{Data availability}

The numerical data that support the findings of this study are available from the corresponding author upon request.

\section{Code availability}

The QDYN library for quantum dynamics and control in Fortran was used for all calculations of the system dynamics and optimization using Krotov's method. It is available upon request through https://www.qdyn-library.net. The Nelder-Mead simplex optimizations were performed using the SciPy Python library (v0.17), freely available at https://scipy.org.

\section{ACKNOWLEDGEMENTS}

We thank the Kavli Institute for Theoretical Physics for their hospitality and for supporting this research in part by the National Science Foundation Grant No. PHY1125915. Financial support by the DAAD under Grant No. PPP USA 54367416 and the National Science Foundation under the Catalyzing International Collaborations program (Grant No. OISE-1158954) is gratefully acknowledged. C.P.K. was also supported by the DFG project "Control of open systems", Grant No. KO 2301/11-1. Publication made possible in part by support from the Berkeley Research Impact Initiative (BRII) sponsored by the UC Berkeley Library.

\section{AUTHOR CONTRIBUTIONS}

M.H.G. performed the numerical calculations and data analysis. All authors contributed to the planning, discussion of results, and preparation of the manuscript.

\section{ADDITIONAL INFORMATION}

Supplementary Information accompanies the paper on the npj Quantum Information website (doi:10.1038/s41534-017-0036-0).

Competing interests: The authors declare no competing financial interests.

Publisher's note: Springer Nature remains neutral with regard to jurisdictional claims in published maps and institutional affiliations.

\section{REFERENCES}

1. Caneva, T. et al. Optimal control at the quantum speed limit. Phys. Rev. Lett. 103, 240501 (2009)

2. Goerz, M. H., Calarco, T. \& Koch, C. P. The quantum speed limit of optimal controlled phasegates for trapped neutral atoms. J. Phys. B 44, 154011 (2011).

3. Sørensen, J. J. W. H. et al. Exploring the quantum speed limit with computer games. Nature 532, 210-213 (2016).

4. Glaser, S. J. et al. Training Schrödinger's cat: quantum optimal control. Eur. Phys. J. D 69, 279 (2015).

5. Koch, C. P. Controlling open quantum systems: tools, achievements, and limitations. J. Phys. Condens. Matter 28, 213001 (2016).

6. van Frank, S. et al. Interferometry with non-classical motional states of a BoseEinstein condensate. Nat. Commun 5, 4009 (2014).

7. Dolde, F. et al. High-fidelity spin entanglement using optimal control. Nat. Commun 5, 3371 (2014).

8. Waldherr, G. et al. Quantum error correction in a solid-state hybrid spin register. Nature 506, 204-207 (2014).

9. Koch, J. et al. Charge-insensitive qubit design derived from the Cooper pair box. Phys. Rev. A. 76, 042319 (2007).

10. Blais, A. et al. Quantum-information processing with circuit quantum electrodynamics. Phys. Rev. A. 75, 032329 (2007).

11. Leek, P. J. et al. Using sideband transitions for two-qubit operations in superconducting circuits. Phys. Rev. B 79, 180511 (2009).

12. Chow, J. M. et al. Simple all-microwave entangling gate for fixed-frequency superconducting qubits. Phys. Rev. Lett. 107, 080502 (2011).

13. Chow, J. M. et al. Universal quantum gate set approaching fault-tolerant thresholds with superconducting qubits. Phys. Rev. Lett. 109, 060501 (2012).

14. Poletto, S. et al. Entanglement of two superconducting qubits in a waveguide cavity via monochromatic two-photon excitation. Phys. Rev. Lett. 109, 240505 (2012).
15. Chow, J. M. et al. Microwave-activated conditional-phase gate for superconducting qubits. New J. Phys. 15, 115012 (2013).

16. Chow, J. M. et al. Implementing a strand of a scalable fault-tolerant quantum computing fabric. Nat. Commun 5, 4015 (2014).

17. Corcoles, A. D. et al. Demonstration of a quantum error detection code using a square lattice of four superconducting qubits. Nat. Commun 6, 7979 (2015).

18. Economou, S. E. \& Barnes, E. Analytical approach to swift nonleaky entangling gates in superconducting qubits. Phys. Rev. B 91, 161405 (2015).

19. Cross, A. W. \& Gambetta, J. M. Optimized pulse shapes for a resonator-induced phase gate. Phys. Rev. A. 91, 032325 (2015).

20. Sheldon, S., Magesan, E., Chow, J. M. \& Gambetta, J. M. Procedure for systematically tuning up cross-talk in the cross-resonance gate. Phys. Rev. A. 93, 060302 (2016).

21. DiCarlo, L. et al. Demonstration of two-qubit algorithms with a superconducting quantum processor. Nature 460, 240-244 (2009).

22. Ghosh, J. et al. High-fidelity controlled- $\sigma^{7}$ gate for resonator-based superconducting quantum computers. Phys. Rev. A. 87, 022309 (2013).

23. Egger, D. J. \& Wilhelm, F. K. Optimized controlled-z gates for two superconducting qubits coupled through a resonator. Supercond. Sci. Technol. 27 014001 (2014).

24. Wallquist, M., Shumeiko, V. S. \& Wendin, G. Selective coupling of superconducting charge qubits mediated by a tunable stripline cavity. Phys. Rev. B 74, 224506 (2006).

25. Srinivasan, S. J., Hoffman, A. J., Gambetta, J. M. \& Houck, A. A. Tunable coupling in circuit quantum electrodynamics using a superconducting charge qubit with a $V$ -shaped energy level diagram. Phys. Rev. Lett. 106, 083601 (2011).

26. Allman, M. S. et al. Tunable resonant and nonresonant interactions between a phase qubit and LC resonator. Phys. Rev. Lett. 112, 123601 (2014).

27. Andersen, C. K. \& Mølmer, K. Multifrequency modes in superconducting resonators: bridging frequency gaps in off-resonant couplings. Phys. Rev. A. 91 023828 (2015).

28. McKay, D. C. et al. Universal gate for fixed-frequency qubits via a tunable bus. Phys. Rev. Appl. 6, 064007 (2016).

29. Martinis, J. M. et al. Decoherence in Josephson qubits from dielectric loss. Phys. Rev. Lett. 95, 210503 (2005).

30. Constantin, M. \& Yu, C. C. Microscopic model of critical current noise in Josephson junctions. Phys. Rev. Lett. 99, 207001 (2007).

31. Motzoi, F. \& Wilhelm, F. K. Improving frequency selection of driven pulses using derivative-based transition suppression. Phys. Rev. A. 88, 062318 (2013).

32. Motzoi, F., Gambetta, J., Rebentrost, P. \& Wilhelm, F. Simple pulses for elimination of leakage in weakly nonlinear qubits. Phys. Rev. Lett. 103, 110501 (2009).

33. Rebentrost, P., Serban, I., Schulte-Herbrüggen, T. \& Wilhelm, F. K. Optimal control of a qubit coupled to a non-Markovian environment. Phys. Rev. Lett. 102, 090401 (2009).

34. Chow, J. M. et al. Optimized driving of superconducting artificial atoms for improved singlequbit gates. Phys. Rev. A. 82, 040305 (2010).

35. Egger, D. J. \& Wilhelm, F. K. Adaptive hybrid optimal quantum control for imprecisely characterized systems. Phys. Rev. Lett. 112, 240503 (2014).

36. Zahedinejad, E., Ghosh, J. \& Sanders, B. C. High-fidelity single-shot Toffoli gate via quantum control. Phys. Rev. Lett. 114, 200502 (2015).

37. Theis, L. S., Motzoi, F. \& Wilhelm, F. K. Simultaneous gates in frequency-crowded multilevel systems using fast, robust, analytic control shapes. Phys. Rev. A. 93, 012324 (2016).

38. Nielsen, M. \& Chuang, I. L. Quantum Computation and Quantum Information (Cambridge University Press, 2000).

39. Chen, Y. et al. Qubit architecture with high coherence and fast tunable coupling Phys. Rev. Lett. 113, 220502 (2014).

40. Schuster, D. I. et al. Resolving photon number states in a superconducting circuit. Nature 445, 515-518 (2007).

41. Blais, A. et al. Cavity quantum electrodynamics for superconducting electrical circuits: An architecture for quantum computation. Phys. Rev. A. 69, 062320 (2004).

42. Rezakhani, A. T. Characterization of two-qubit perfect entanglers. Phys. Rev. A. 70 052313 (2004)

43. Motzoi, F., Gambetta, J. M., Merkel, S. T. \& Wilhelm, F. K. Optimal control methods for rapidly time-varying Hamiltonians. Phys. Rev. A. 84, 022307 (2011).

44. Jäger, G., Reich, D. M., Goerz, M. H., Koch, C. P. \& Hohenester, U. Optimal quantum control of bose-einstein condensates in magnetic microtraps: Comparison of grape and krotov optimization schemes. Phys. Rev. A. 90, 033628 (2014).

45. Raftery, J. et al. Direct digital synthesis of microwave waveforms for quantum computing, arXiv:1703.00942 (2017)

46. Goerz, M. H., Halperin, E. J., Aytac, J. M., Koch, C. P. \& Whaley, K. B. Robustness of high-fidelity Rydberg gates with single-site addressability. Phys. Rev. A. 90, 032329 (2014). 
47. Dong, D., Chen, C., Qi, B., Petersen, I. R. \& Nori, F. Robust manipulation of superconducting qubits in the presence of uctuations. Sci. Rep. 5, 7873 (2015).

48. Allen, J. L., Kosut, R., Joo, J., Leek, P. \& Ginossar, E. Optimal control of two qubits via a single cavity drive in circuit quantum electrodynamics. Phys. Rev. A. 95, 042325 (2017).

49. Goerz, M. H., Whaley, K. B. \& Koch, C. P. Hybrid optimization schemes for quantum control. EPJ Quantum Technol 2, 21 (2015).

50. Reich, D. M., Ndong, M. \& Koch, C. P. Monotonically convergent optimization in quantum control using Krotov's method. J. Chem. Phys. 136, 104103 (2012).

51. Müller, M. M. et al. Optimizing entangling quantum gates for physical systems. Phys. Rev. A. 84, 042315 (2011)

52. Makhlin, Y. Nonlocal properties of two-qubit gates and mixed states, and the optimization of quantum computations. Quantum Inf. Process 1, 243-252 (2002).

53. Zhang, J., Vala, J., Sastry, S. \& Whaley, K. B. Geometric theory of nonlocal twoqubit operations. Phys. Rev. A. 67, 042313 (2003).

54. Watts, P. et al. Optimizing for an arbitrary perfect entangler: I. Functionals. Phys. Rev. A. 91, 062306 (2015).

55. Pedersen, L. H., Møller, N. M. \& Mølmer, K. Fidelity of quantum operations. Phys. Lett. A. 367, 47-51 (2007).
56. Tal-Ezer, H. On restart and error estimation for Krylov approximation of $w=f(a) v$. SIAM J. Sci. Comput. 29, 2426-2441 (2007).

57. Peterer, M. J. et al. Coherence and decay of higher energy levels of a superconducting transmon qubit. Phys. Rev. Lett. 114, 010501 (2015).

Open Access This article is licensed under a Creative Commons Attribution 4.0 International License, which permits use, sharing, adaptation, distribution and reproduction in any medium or format, as long as you give appropriate credit to the original author(s) and the source, provide a link to the Creative Commons license, and indicate if changes were made. The images or other third party material in this article are included in the article's Creative Commons license, unless indicated otherwise in a credit line to the material. If material is not included in the article's Creative Commons license and your intended use is not permitted by statutory regulation or exceeds the permitted use, you will need to obtain permission directly from the copyright holder. To view a copy of this license, visit http://creativecommons. org/licenses/by/4.0/.

(c) The Author(s) 2017 